\title{
Coherent electron-nuclear coupling in oligothiophene molecular wires
}

\author{
Jascha Repp ${ }^{1,2 \star}$, Peter Liljeroth ${ }^{1,3}$ and Gerhard Meyer ${ }^{1}$
}

In molecular electronics individual molecules serve as electronic devices. In these systems, electron-vibron (e-v) coupling can be expected to lead to new physical phenomena and potential device functions ${ }^{1-3}$. In previous studies of molecular wires, the $e-v$ coupling occurred as a result of the well-known Franck-Condon principle, for which the Born-Oppenheimer approximation holds. This means that after a vibronic excitation, the electrons and the vibrations evolve independently from each other. Here we show that this simple picture changes markedly when two electronic levels in a molecule are coupled by a molecular vibration ${ }^{4,5}$. In molecular wires we observe a non-Born-Oppenheimer regime, for which a coherent coupling of electronic and nuclear motion emerges $^{6}$. This phenomenon should occur in all systems with strong electron-vibration coupling and an electronic level spacing of the order of vibrational energies. The coherent coupling of electronic and nuclear motion could be used to implement mechanical control of electron transport in molecular electronics.

In recent years, chains of metal atoms on surfaces have attracted considerable attention ${ }^{7-9}$. These studies concentrated on the evolution of the electronic structure from individual atoms to long chains, enabling the visualization of individual particle-ina-box states ${ }^{7,8}$. Most recently, the off-resonance conductance ${ }^{10,11}$, dispersion of occupied states ${ }^{12}$ and reactivity ${ }^{13}$ of molecular analogues to such atomic chains were studied. Conjugated molecular chains are relevant both as molecular wires in molecular electronics ${ }^{14,15}$ and as constituents in organic electronic devices. In contrast to metal chains, strong electron-vibron (e-v) coupling can be expected to play an important role ${ }^{16,17}$ leading to new physical phenomena and possible device functions ${ }^{1-3}$.

In most molecular systems studied so far, $e-v$ coupling arises as a consequence of the instantaneous electron attachment associated with a tunnelling process that is much faster than nuclear reorganization. This regime can be understood from the well-known Franck-Condon principle, for which the Born-Oppenheimer approximation holds. This means that after the tunnelling process, the electrons and the vibrations evolve independently from each other.

In this Letter, we show that this simple picture is markedly changed when two electronic levels in a molecule are coupled by a molecular vibration ${ }^{4,5}$. A non-Born-Oppenheimer regime occurs in molecular wires based on oligothiophene molecules, which we studied by low-temperature scanning tunnelling microscopy (STM) and spectroscopy as a function of their chain length. For the longest chains, we observe coherent coupling of electronic and nuclear motion indicating a complete breakdown of the Born-Oppenheimer approximation ${ }^{6}$.
Oligothiophene molecules were studied on ultrathin insulating films $(\mathrm{NaCl}, \mathrm{RbI}$ and $\mathrm{Xe})$ on copper single crystals by low-temperature STM operated at $T=5 \mathrm{~K}$. For further details see Supplementary Information. At positive voltages below the first molecular resonance (lowest unoccupied molecular orbital, LUMO), the molecules appear as wire-like long protrusions (see Fig. 1a). We used differential conductance $(\mathrm{d} I / \mathrm{d} V)$ spectroscopy to identify the energetic positions of the molecular resonances at positive voltage polarity (Fig. 1b). We can map the spatial dependence of these orbitals by bias-dependent imaging at voltages corresponding to the resonances ${ }^{18}$. Figure $1 \mathrm{c}$ was acquired at $0.8 \mathrm{~V}$, below the first resonance, and reflects the geometry of the molecule. Figure 1d shows the LUMO, which has a single lobe with a maximum at the centre of the molecule. Only at the very ends are there two nodal planes, and two small lobes having an orbital density of opposite sign stick out. The schematic orbital structure of the LUMO of oligothiophene is shown in Fig. 1e (ref. 19). For an oligothiophene with six units, this leads to the orbital structure shown in Fig. 1f. Note the additional nodal plane in the molecular plane that is not visible in the top view. In our experiments, the orbital structure is probed at very low densities because of the low currents used. Far outside the molecule, the two outer lobes merge and hide the inner lobe of opposite sign; the latter sticks out only at the ends of the molecule (Fig. $1 \mathrm{~g}$ ). This is reproduced by the experimental LUMO image in Fig. 1d. The subsequent orbitals $(\mathrm{LUMO}+1, \mathrm{LUMO}+2$ and so on) all derive from the LUMO of the subunits, and their symmetries can be well understood by considering one-dimensional-confined (1D-confined) states. As we go from $n_{\mathrm{e}}$ to $n_{\mathrm{e}}+1$, the number of nodes along the molecular axis increases by one, where $n_{\mathrm{e}}$ refers to the number of the state. This can be seen in the corresponding STM images, which are shown at high contrast in Fig. 2a-e.

To obtain a systematic understanding of the energetic positions of the resonances as a function of the oligothiophene chain length $l$, we carried out spectroscopic experiments on a large number of oligothiophene molecules adsorbed on $\mathrm{NaCl}$ bilayers on $\mathrm{Cu}(111)$. This yields the energy levels as a function of $l$ (Fig. 2f) for the unoccupied molecular orbitals. As expected, the molecular resonances decrease in energy for increasing chain length. The data can be fitted by a $1 / l$ behaviour (see Fig. $2 \mathrm{f}$ ).

In a simple picture of $1 \mathrm{D}$-confined states, the energies would only be a function of the corresponding wavenumber $k=n_{\mathrm{e}} \pi / l$ (refs 7,20,21), where $n_{\mathrm{e}}$ is the number of the $1 \mathrm{D}$ state. However, it is important to note that, in contrast to the situation of chains of metal atoms on metals, we temporarily add an electron to the system when tunnelling into one of the resonances. Therefore, the spectra reflect the negative ion resonance spectra of the molecule, which involves considerable Coulomb energies owing to the addition of

\footnotetext{
${ }^{1}$ IBM Research - Zurich, 8803 Rüschlikon, Switzerland, ${ }^{2}$ Institute of Experimental and Applied Physics, University of Regensburg, 93040 Regensburg, Germany, ${ }^{3}$ Debye Institute for Nanomaterials Science, University of Utrecht, PO Box 80000, 3508TA Utrecht, The Netherlands.

*e-mail: jascha.repp@physik.uni-regensburg.de.
} 


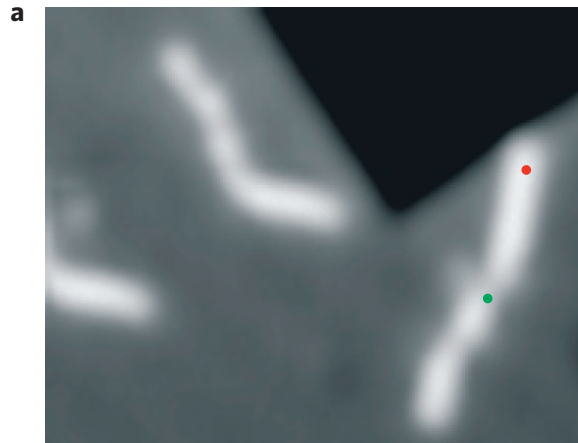

b

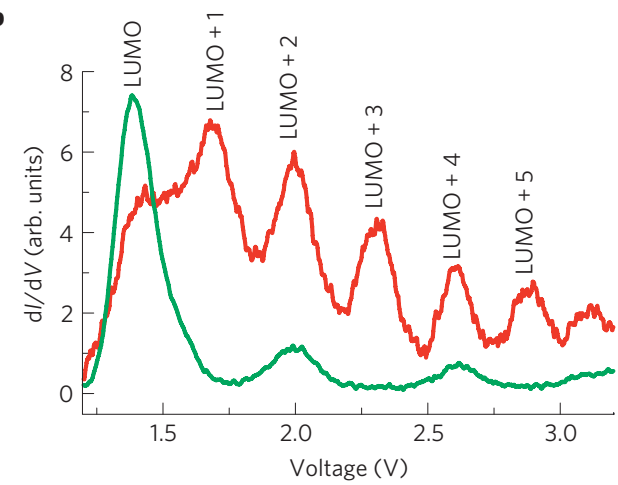

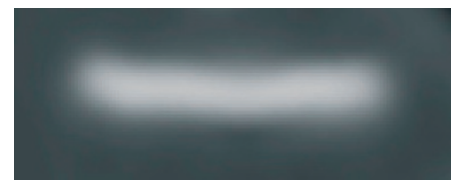
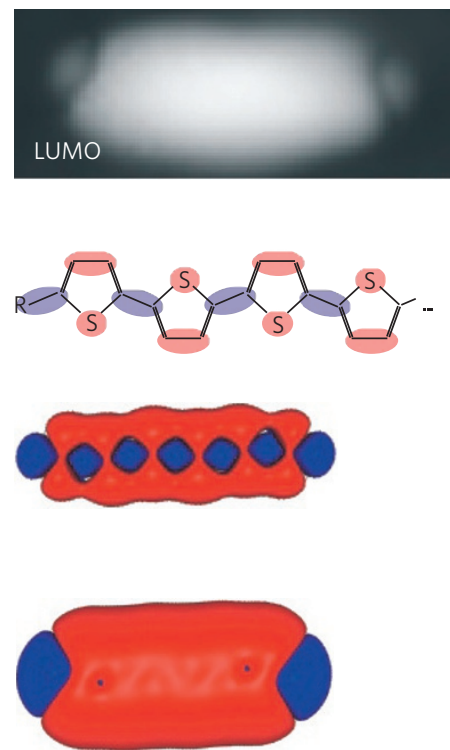

Figure 1 | Structure model and bias-dependent STM images of thiophene oligomers measured on a NaCl bilayer on Cu(111). a, In-gap STM image of three oligomers showing their orientation. $100 \AA \times 80 \AA ; 1.3 \mathrm{~V} ; 0.35 \mathrm{pA}$. b, dl/dV spectra measured in two different spots over one of the oligothiophene molecules as indicated in a. In the centre of the molecule (green), the LUMO peak is very pronounced and at the end of the molecule (red) higher-lying states can be detected. c,d, In-gap and LUMO images of one straight thiophene molecule (length: $44 \AA$ ). $65 \AA$ × $40 \AA$; $0.8 \mathrm{~V} ; 0.5$ pA and $1.5 \mathrm{~V}$; 0.25 pA. e, Schematic drawing of the molecular structure and LUMO orbital. f,g, Calculated LUMO orbital of an oligothiophene molecule (length: 6 monomer units) at moderately and very low orbital density.

an electron, which will depend on the length of the molecule. We plot the dispersion in Fig. $2 \mathrm{~g}$ from the states of the longest molecular wire alone (rightmost column of points in Fig. 2f). In this case, in good approximation, the Coulomb and the polarization energies give only a constant shift of all of the points, but do not modify the functional dependence of $E$ on $k$. Surprisingly, the dispersion curve is not parabolic for small wavenumbers as would be expected from both particle-in-box and simple tight-binding descriptions. Instead, the dispersion can be well fitted by a linear dispersion with a slope of $4.9 \mathrm{eV} \AA$ down to the smallest wavenumber. For shorter oligomers we also obtain linear dispersion curves as can be seen from the points in each column in Fig. $2 \mathrm{f}$ being equidistant. The dispersion relation is independent of the underlying thin insulating film as we obtain the same behaviour and slope for both $\mathrm{NaCl}$ and $\mathrm{RbI}$ films. Only the absolute energies change in accordance with the difference in the workfunctions of the different substrate systems. Density functional theory calculations ${ }^{19}$ suggest a dispersion that is linear for higher wavenumbers but becomes parabolic close to $k=0$ (see Supplementary Information). This agrees with the experimental results at higher $k$, but cannot account for the observed linear dispersion down to small values of $k$.

As shown previously, the molecular spectra have a significantly lower broadening on $\mathrm{RbI}$ and $\mathrm{Xe}$ films than on $\mathrm{NaCl}$ (refs 22, 23). We exploit this to resolve the vibronic features ${ }^{24}$ in the $\mathrm{d} I / \mathrm{d} V$ spectra of the oligothiophene. If the Born-Oppenheimer approximation is valid, an electron injected into a molecule at an excess energy above the energy of an electronic resonance can excite a molecular vibration with exactly that energy. In $\mathrm{d} I / \mathrm{d} V$ spectroscopy in a double-barrier tunnel junction geometry, this results in satellite peaks beyond an electronic resonance with the energy separation of $h v$ (refs 25-27). As can be seen in Fig. 3c,d, the two most intense peaks can be assigned to well-defined electronic levels and are followed by two satellite peaks at approximately 180 and $2 \times 180 \mathrm{meV}$ higher energy. This energy lies in the range of the aromatic $\mathrm{C}-\mathrm{C}$-stretch vibrations, which are expected and observed to couple strongly to electrons on the delocalized molecular orbitals ${ }^{28}$. The intensity of the first vibronic satellite is about three times smaller than the elastic peak in the tunnelling spectra ${ }^{25}$. This ratio equals the dimensionless parameter of the e- $v$ coupling strength and the large difference in intensity allows for an easy discrimination between elastic and vibronic satellite peaks. We will indicate the vibronic states by two quantum numbers. The first will refer to the number $n_{\mathrm{e}}$ of the electronic 1D-confined state. The second refers to the number of vibrons (only one relevant mode is considered, see Supplementary Information) excited in the molecule. For example, $\Psi=|1,0\rangle$ denotes the LUMO with no excited vibrons. The above e- $v$ coupling is consistent with the Franck-Condon picture, for which the Born-Oppenheimer approximation holds. The corresponding state factorizes into an electronic and a vibrational contribution as $\Psi=\Psi_{\mathrm{e}} \cdot \Psi_{v}$. In our experiments this regime applies to the short molecular wires.

This simple picture is markedly changed when two or more electronic levels in a molecule are coupled by a suitable molecular vibration $^{4,5}$. If, for example, the separation between the LUMO and the LUMO +1 is similar to the energy of a vibration, the states $|1,1\rangle$ and $|2,0\rangle$ will be at similar energy. The two states will then interact resulting in an avoided crossing, at which the energy separation will be approximately $\Delta E=2 \gamma$ (refs 4,5), determined by the e $-v$ coupling term $\gamma$. For these resulting states the Born-Oppenheimer approximation breaks down completely and vibrational and electronic states are coupled coherently ${ }^{6}$. These 
a

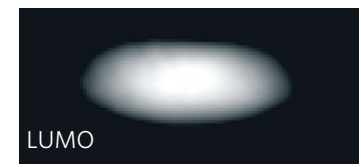

b

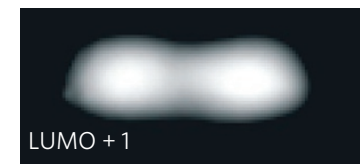

c

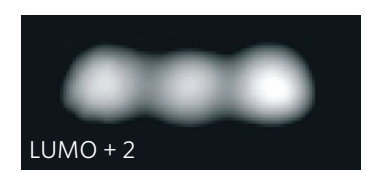

d

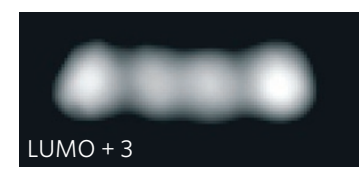

e

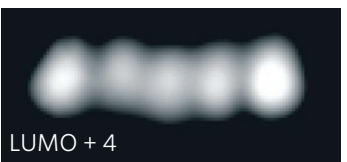

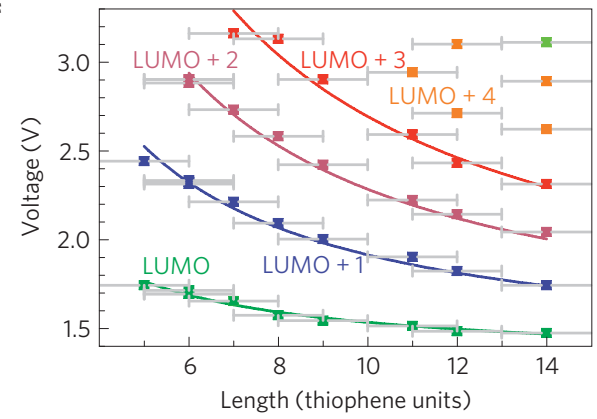

g

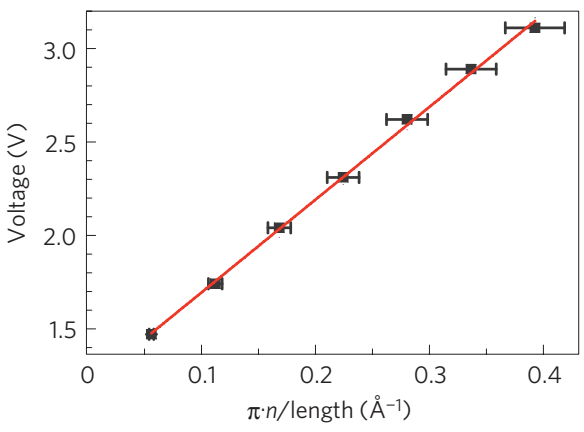

Figure 2 | Electronic level structure of oligothiophene measured on a $\mathrm{NaCl}$ bilayer on $\mathrm{Cu}(111)$. a-e, STM images that represent the consecutive molecular orbitals, starting with the LUMO of the same molecule as imaged in Fig. 1c,d. Bias voltages (a-e): 1.5 V, 1.9 V, 2.3 V, 2.6 V and 3.0 V, set-point 0.25 pA. f, Measured orbital energies (symbols) as a function of the oligothiophene length $\mathrm{I}$. The solid lines are fits to $1 / \mathrm{l}$ behaviour. $\mathbf{g}$, Dispersion curve of oligothiophene and a fit to a linear dispersion (solid line) having a slope of $4.9 \mathrm{eV} \AA$. $n$ refers to the number of the state $(n=1$ for the LUMO). Molecule length is approximately $56 \AA$. All error bars refer to the uncertainty in the lengths of the molecules, which was inferred from the in-gap STM images. Repeated measurements of the same molecular length yielded values differing by at most one monomer unit ( $\pm 4 \AA$ ) owing to their bent adsorption geometry.

non-Born-Oppenheimer states can no longer be factorized into an electronic and a vibrational contribution.

This regime of mixed vibronic states can be accessed experimentally by tuning the energies of the electronic states of oligothiophene through their length dependence. For sufficiently long molecular chains, the spacing between LUMO and LUMO +1 becomes similar to the vibrational energies. For the following analysis we eliminated the absolute energy scale by plotting all levels relative to the lowest state (with $\Delta E=0$ ). To clearly identify the signatures indicative for the breakdown of the Born-Oppenheimer approximation in our experiments, we first discuss the expected behaviour as plotted in Fig. 3a with and without the coherent $e-v$ coupling. The energy differences of the electronic states are derived from density functional theory calculations of the free molecule (see Supplementary Information). Without $\mathrm{e}-\boldsymbol{v}$ coupling (dashed lines), the vibronic state of the LUMO with one vibrational quantum excited $(|1,1\rangle)$ is constantly higher in energy by $\Delta E=h v$ and crosses the LUMO +1 $(|2,0\rangle)$ state. Note that in this case, the spatial density of the electronic part of the states $(|1,1\rangle)$ is exactly that of the LUMO $(|1,0\rangle)$, which is indicated by colouring the lines blue for the LUMO and red for LUMO +1 . When including $e-v$ coupling (solid lines), the behaviour changes markedly. Now, $|1,1\rangle$ and $|2,0\rangle$ exhibit an avoided crossing: whereas for large values of $k$ they are close to their original identity $|1,1\rangle$ and $|2,0\rangle$, near the avoided crossing they are a coherent superposition of those two states ${ }^{6}$. This is the signature of the breakdown of the Born-Oppenheimer approximation. Whereas the values in Fig. 3a are derived from a simple numerical diagonalization of the e- $v$ Hamiltonian (see Supplementary Information), the general features of the avoided crossing can be rationalized irrespectively of the details of the simulations ${ }^{4,5}$. The most important parameters are the vibrational energy quantum $h v$ and the e- $v$ coupling strength, which can both be extracted from the experimentally observed vibronic satellites for short molecular wires.
The experimental values were obtained from the molecular wires on a Xe monolayer. Again, all levels are plotted relative to the lowest state, which also removes the Coulomb term owing to the temporary charging of the molecule. Second, we plot the energies as a function of the energy separation between two specific states (instead of the length of the molecule). For short molecular wires, these two states correspond to the LUMO and LUMO +1 . This way we remove the uncertainty associated with the determination of the molecular wire length (see the error bars in Fig. 2). This results in only a slight rescale of the abscissa in the resulting graph shown in Fig. $3 \mathrm{~b}$ as compared with Fig. 3a. For easy reference the approximate lengths of the molecules are given at the top of the graph. In Fig. $3 \mathrm{~b}$ experimental data are shown as dots and calculated values as grey lines. The black and blue dots refer to the lowest state and hence to $E=0$ (black) and to our reference state (blue) used to calculate the level spacing. Thus, these dots do not contain independent information. The quantitative agreement between theory and experiment is evident. Most importantly, in comparing Fig. $3 \mathrm{~b}$ to $\mathrm{a}$, the qualitative signatures of the avoided crossing are clearly visible. Note that in the important region of the avoided crossing the reference state (blue) is always the third lowest state and is not an arbitrary choice. In other words, all of the analysed molecules showed two distinct resonances for the anticrossing states that never come closer to each other than $0.1 \mathrm{~V}$.

Further experimental evidence for the avoided crossing comes from the peak intensities and the spatial distribution of the anticrossing states. Directly at the avoided crossing of the second and third lowest states, they should be of the form $(|1,1\rangle+|2,0\rangle) / \sqrt{2}$ and $(|1,1\rangle-|2,0\rangle) / \sqrt{2}$. As STM probes the electronic part of the wavefunction, the spatial dependence of both these coherently coupled states should be a superposition of the LUMO and the $\mathrm{LUMO}+1$. For symmetry reasons, their peak intensities should also be similar. When going from short to long molecules, the second 


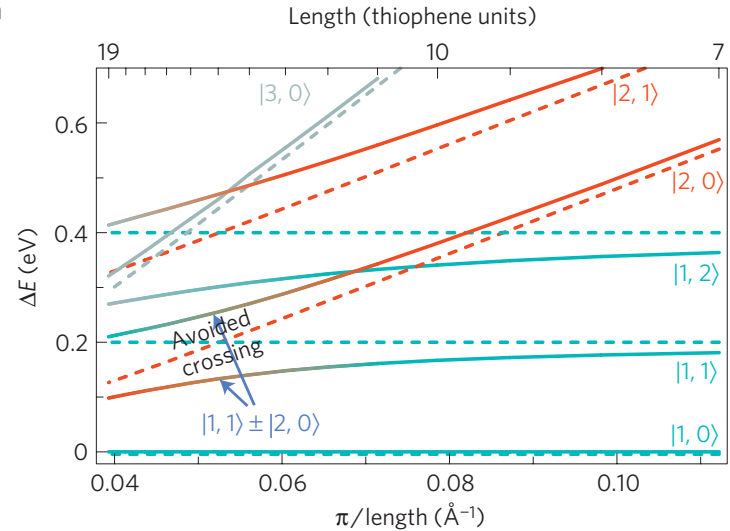

b

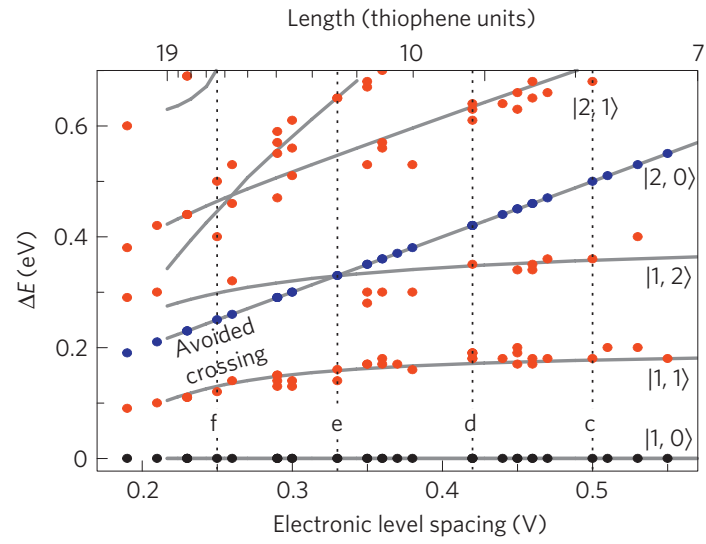

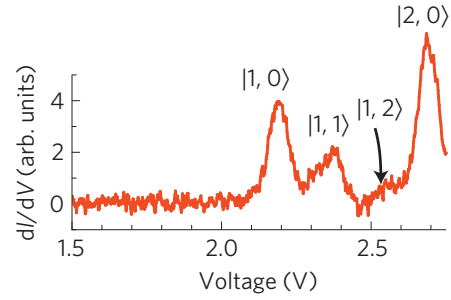

d
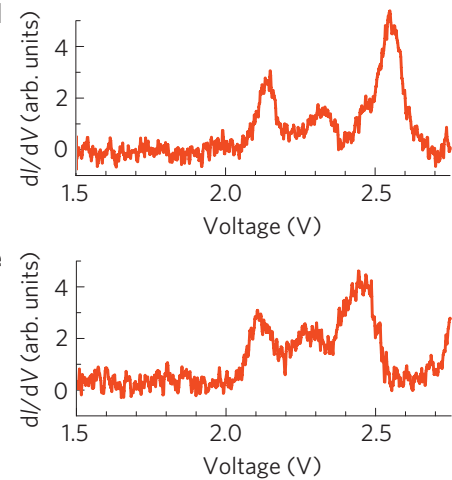

f

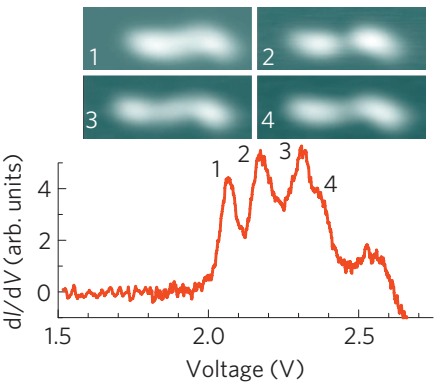

Figure 3 | Transition in the vibronic structure from separate electronic and vibronic levels to strongly coupled non-Born-Oppenheimer levels.

a, Calculated vibronic levels as a function of inverse chain length excluding (dashed lines) and including (solid lines) e- $v$ coupling. The colours indicate the nature of the electronic part of the wavefunction: blue denotes LUMO, red LUMO +1 and grey other. $\mathbf{b}$, Measured (dots) and calculated (lines) vibronic levels as a function of the spacing between two specific levels (blue and black). In $\mathbf{a}$ and $\mathbf{b}$ the lowest level was taken as zero reference ( $\Delta E=0$ ). The molecular lengths given at the top of the graphs refer to the calculated values. c-f, Experimental $\mathrm{d} / \mathrm{d} V$ spectra showing the transition in the vibronic structure from short to long oligomers measured on a Xe monolayer on $\mathrm{Cu}(111)$. The data points in $\mathbf{b}$ corresponding to these spectra are marked by vertical dashed lines. $\mathbf{f}$ shows in addition the corresponding constant-height spatially resolved $\mathrm{d} / \mathrm{d} V$ maps for the first four peaks in the spectrum.

lowest state should develop continuously from a LUMO character to a LUMO + 1 character. The lowest state should be mostly LUMO in nature, but should exhibit a slight deviation from LUMO for very long chains because of the admixture of other states. All of these features can be observed in the evolution of these states as a function of the oligothiophene chain length shown in Fig. 3c-f (see also Supplementary Information). Most importantly, close to the avoided crossing (Fig. 3f) the three lowest states show similar intensities, which cannot be reconciled with the Born-Oppenheimer regime, in which one state would be the satellite of another one. Thus, this is another indication for the non-Born-Oppenheimer regime.

We note that this effect will also lead to an apparent linearization of the dispersion. In our simple calculation the lowest state of the longest chains (yielding the smallest values of $k$ ) is decreased by approximately $25 \mathrm{meV}$ as a result of an admixture of other states (pseudo Jahn-Teller effect) ${ }^{24}$. Moreover, it increases the energy separation between the lowest state and the third lowest state that would be misinterpreted as being purely electronic in nature $(\mathrm{LUMO}+1)$ not taking the Born-Oppenheimer breakdown into account.

But what is the nature of the correlated electronic and vibrational states in our case? We discuss here the simplest case directly at the avoided crossing, where the electronic level spacing exactly matches $h v$. The semi-classical analogue would be the electromechanical oscillator: the electron oscillates between the left and the right of the molecule at the oscillation period of the vibration (Rabi oscillation). The vibration and the movement of the electron have the same frequency because of $\varepsilon_{\mathrm{LUMO}+1}-\varepsilon_{\mathrm{LUMO}}=h v$, giving rise to a collective and coherent motion of the electron and the nuclei (for details, see Supplementary Information).

These findings have important implications for organic as well as molecular electronics. The prerequisite for the effects observed here is only the coupling of two electronic levels with an energy separation of approximately $h v$ by one molecular vibration with frequency $v$. Large conducting molecules have a dense electronic spectrum, so that this condition will be fulfilled in many cases of practical importance ${ }^{16,17}$. As a result of the strong coupling, this effect leads to very strong shifts of resonances. Even far away from the resonant case, it results in vibronic satellites that deviate significantly from the original $h v$ energy spacing ${ }^{29,30}$. Thus, the incorporation of such electronic-nuclear coupling into models is important for understanding molecular wires. Apart from being of fundamental interest, coherent electron-nuclear coupling may open up new physics for future molecular devices, as it allows for a mechanical control of electronic properties of the molecule.

Received 18 May 2010; accepted 31 August 2010; published online 17 October 2010 


\section{References}

1. Galperin, M, Ratner, M. A., Nitzan, A. \& Troisi, A. Nuclear coupling and polarization in molecular transport junctions: Beyond tunnelling to function. Science 319, 1056-1060 (2008).

2. Heeger, A. J., Kivelson, S., Schrieffer, J. R. \& Su, W-P. Solitons in conducting polymers. Rev. Mod. Phys. 60, 781-850 (1988).

3. Galperin, M., Ratner, M. A. \& Nitzan, A. Molecular transport junctions: Vibrational effects. J. Phys. Condens. Matter 19, 103201 (2007).

4. Brawer, S. Dynamical effects on nearly degenerate electronic levels in solids. J. Chem. Phys. 68, 3352-3359 (1978).

5. Malta, O. L. The theory of vibronic transitions in rare earth compounds. J. Phys. Chem. Solids 56, 1053-1062 (1995).

6. Reckermann, F., Leijnse, M., Wegewijs, M. R. \& Schoeller, H. Transport signature of pseudo Jahn-Teller dynamics in a single-molecule transistor. Europhys. Lett. 83, 58001 (2008).

7. Nilius, N., Wallis, T. M. \& Ho, W. Development of one-dimensional band structure in artificial gold chains. Science 297, 1853-1856 (2002).

8. Crain, J. N. \& Pierce, D. T. End states in one-dimensional atom chains. Science 307, 703-706 (2005).

9. Hirjibehedin, C. F., Lutz, C. P. \& Heinrich, A. J. Spin coupling in engineered atomic structures. Science 312, 1021-1024 (2006).

10. Choi, S. H., Kim, B. \& Frisbie, C. D. Electrical resistance of long conjugated molecular wires. Science 320, 1482-1486 (2008).

11. Lafferentz, L. et al. Conductance of a single conjugated polymer as a continuous function of its length. Science 323, 1193-1197 (2009).

12. Koller, G. et al. Intra- and intermolecular band dispersion in an organic crystal. Science 317, 351-355 (2007).

13. Maksymovych, P., Sorescu, D. C., Jordan, K. D. \& Yates, J. T. Collective reactivity of molecular chains self-assembled on a surface. Science 322, 1664-1667 (2008).

14. Moth-Poulsen, K. \& Bjørnholm, T. Molecular electronics with single molecules in solid-state devices. Nature Nanotechnol. 4, 551-556 (2009).

15. Nitzan, A. \& Ratner, M. A. Electron transport in molecular wire junctions. Science 300, 1384-1389 (2003).

16. LeRoy, B. J., Lemay, S. G., Kong, J. \& Dekker, C. Electrical generation and absorption of phonons in carbon nanotubes. Nature 432, 371-374 (2004).

17. Leturcq, R. et al. Franck-Condon blockade in suspended carbon nanotube quantum dots. Nature Phys. 5, 327-331 (2009).

18. Repp, J., Meyer, G., Stojkovic, S. M., Gourdon, A. \& Joachim, C. Molecules on insulating films: Scanning-tunnelling microscopy imaging of individual molecular orbitals. Phys. Rev. Lett. 94, 026803 (2005).

19. Telesca, R. et al. Density-functional study of the evolution of the electronic structure of oligomers of thiophene: Towards a model Hamiltonian. Phys. Rev. B 63, 155112 (2001).
20. Wallis, T. M., Nilius, N. \& Ho, W. Electronic density oscillations in gold atomic chains assembled atom by atom. Phys. Rev. Lett. 89, 236802 (2002).

21. Fölsch, S., Hyldgaard, P., Koch, R. \& Ploog, K. H. Quantum confinement in monatomic $\mathrm{Cu}$ chains on $\mathrm{Cu}(111)$. Phys. Rev. Lett. 92, 056803 (2004).

22. Repp, J., Meyer, G., Paavilainen, S., Olsson, F. E. \& Persson, M. Scanning tunnelling spectroscopy of $\mathrm{Cl}$ vacancies in $\mathrm{NaCl}$ films: Strong electron-phonon coupling in double-barrier tunnelling junctions. Phys. Rev. Lett. 95, 225503 (2005).

23. Liljeroth, P., Repp, J. \& Meyer, G. Current-induced hydrogen tautomerization and conductance switching of naphthalocyanine molecules. Science 317, 1203-1206 (2007).

24. Bersuker, I. B. \& Polinger, V. Z. Vibronic Interactions in Molecules and Crystals (Springer, 1989).

25. Wingreen, N. S., Jacobsen, K. W. \& Wilkins, J. W. Inelastic scattering in resonant tunnelling. Phys. Rev. B 40, 11834-11850 (1989).

26. Qiu, X. H., Nazin, G. V. \& Ho, W. Vibronic states in single molecule electron transport. Phys. Rev. Lett. 92, 206102 (2004).

27. Ogawa, N., Mikaelian, G. \& Ho, W. Spatial variations in submolecular vibronic spectroscopy on a thin insulating film. Phys. Rev. Lett. 98, 166103 (2007).

28. Negri, F. \& Zgierski, M. Z. The vibronic structure of the $S_{0} \leftrightarrow S_{1}$ and $S_{0} \leftrightarrow S_{2}$ transitions in simple oligomers of thiophene. J. Chem. Phys. 100, 2571-2587 (1994).

29. Fulton, R. L. \& Gouterman, M. Vibronic coupling. I. Mathematical treatment for two electronic states. J. Chem. Phys. 35, 1059-1071 (1961).

30. Monturet, S. \& Lorente, N. Inelastic effects in electron transport studied with wave packet propagation. Phys. Rev. B 78, 035445 (2008).

\section{Acknowledgements}

We thank M. Wegewijs, F. Reckermann, D. Ryndyk, K. Richter and R. Allenspach for fruitful discussions. We acknowledge financial support by the Volkswagen Foundation (J.R., Lichtenberg program), DFG (J.R., priority program SPP 1243), NWO / Chemical sciences (P.L., Vidi-grant 700.56.423), EC's project ARTIST (G.M., grant agreement no. 243421) and the Swiss NCCR 'Nanoscale Science' (G.M.).

\section{Author contributions}

J.R. and G.M. conceived and set-up the experiments; J.R. and P.L. carried them out. J.R., P.L. and G.M. analysed the results and wrote the Letter.

\section{Additional information}

The authors declare no competing financial interests. Supplementary information accompanies this paper on www.nature.com/naturephysics. Reprints and permissions information is available online at http://npg.nature.com/reprintsandpermissions. Correspondence and requests for materials should be addressed to J.R. 